Aus dem Landesbad der Rheinprovinz in Aachen.

\title{
Die Beziehungen der Esophylaxie zur physikalischen
} Therapie.

\section{Von Walter Krebs.}

E. Hof $\mathrm{mann}(\text { Bonn })^{2}$ ) hat in einer auBerordentlich lesens. und beachtenswerten Abhandlung "Ueber eine nach innen gerichtete Schutzfunktion der Haut (Esophylaxie) nebst Bemerkungen über die Entstehung der Paralyse" Anschauungen entwickelt, die für die Erklärung der Wirkungsweise einer großen Reihe physikalisch-therapeutischer Anwendungen von großer Bedeutung werden können.

Hoffmann - der bei seinen Darlegungen wiederholt auf die Antrittsvorlesung des Züricher Dermatologen Bloch zurïckgreift begrundet die Vorstellung, daß die menschliche Haut den Körper nicht nur gegen äußere Schädigungen zu bewahren hat, sondern auch eine Schutzfunktion für die inneren Organe besitzt, in eingehenden Darlegungen. Im besonderen fesselt seine Auffassung von der Möglichkeit einer nach innen gerichteten Sekretion der Haut, die er besonders auf die Epithelien der Epidermis bezieht; sie seien es, die zu einer Bildung bzw. Vermehrung von Schutzstoffen geeignet und beizutragen imstande seien.

Wenn man bedenkt, daß alle Zellen des Körpers in einem "regen Wechselverkehr mit den Gewebssäften" - Lymphe und Blut - stehen und daß nicht nur Endprodukte - Schlacken - des Stoffwechsels, sondern auch spezifische Körper, welche in den Zellen

1) Interessante und lehrreiche Ausbllcke, auf die hier nicht elngegangen werden soil, ergeben sich bei Gegenuberstellung von Fieberkurven tei Erkrankungen durch Endotoxin bakterien (Typhus) zu Kurven, die von Erkrankungen durch typische Toxinbildner (Diphtherie)
stammen. -3 D. m. W. $1919 \mathrm{Nr} .45$. 
zum Aufbau gelangen, abgegeben werden, die wieder als Reizstoffe für die Zellen anderer Organe dienen können - so muß es in der Tat fast verwunderlich erscheinen, daß diese Auffassung $H$ off mauns von der erweiterten Funktion der Haut nicht schon längst zum Ausdruck gelangt und entsprechend verwertet worden ist. ${ }^{1}$ )

Seine Gedantien weiterzuspiunen und sie für die Erklärung der Wirkung zahlreicher physikalisch-therapeutischer Anwendungen her anzuziehen, liegt nahe, und ich kann es mir nicht versagen, im Nachfolgenden einige entsprechende Bemerkungen zu machen, die zur Klärung der angeregten Fragen meıner Auffassung nach wohl beizutragen vermögen.

Beginnen wir mit der Hydrotherapie, so ist es gestattet, bereits die sogenannte "Reaktion" durch die neue Auffassung von der er weiterten Hauttätigkeit in ein anderes Licht zu rücken. Unter Reaktion versteht man in der Hydrotherapie eine Gruppe von $\mathrm{Er}$ scheinungen, die nach der Anwendung kalter Wasserprozeduren eintreten müssen, wenn anders der mit der lialten Wasseranwendung beabsichtigte Zweck erziclt werden soll: nach einem anfänglichen Abblassen der Haut müssen seliundäre Rötung und ein gesteigertes Warme-, Erfrischungs- und Kräftegefühl eintreten. Diese Erscheinung erklärte man sıch bisher als eıne Folge der Einwirkung aut das Zirkulations- und Nervensystem und vergais eben dabeı ganz, daß die Haut nicht nur die Tràgerin von Getäßen und Nerven usw. sei, sondern auch ein Organ mit eigenem Leben und eigener Tätigkeit. Sicherlich ist das reaktive Wolilbefinden nach Kaltreizen zu einem wesentlichen Teil durch reflektorische Beeinflussung des Zentralnervensystems bedingt. Ist es aber anderseits bei den mehr minder erheblichen Einwirkungen des Kältereizes, der meist durch mechanische Reize, wie Dusche, Frottierung, Massage usw., noch verstärkt wird, nicht sehr wohl möglich, daß die Haut dadurch selbst zu lebhafter Tätigkeit angeregt wird und Stoffe produziert, die das besagte Erfrischungsgefühl hervorrufen? Haben wir nicht ein Gegenstück dazu gewiscermaßen in dem Auttreten des Ermüdungs gefühls nach Ueberanstrengung, das die Folge einer Anhäufung von Stofiwechselprodukten, namentlich von solcnen unvollkommener Oxydation, darstellt? Denn die Lehre vom Auftreten der Ermüdungs toxine gilt nicht nur für den überanstrengten Muskel, sondern iı gleicher Weise für die ganze lebendige Substanz. (Verworn.)

Diese Auffassung vom Entstehen des Erfrischungsgefuhls durch vermehrte Hautfunktion erhält von der Lehre Weichardts über die Protoplasmaaktivierung eine gewisse Stützung.

W e ichardt betont in seinen Arbeitell, daß dic allgemeine Leistungssteigerung bei parenteraler Einverleibung von Eiweißspaltprodukten als Summe der gesteigerten Leistung der verschiedenen Organsysteme aufzufassen ist. Schon vor Jahren stellte er den Satz der Leistungssteigerung infolge von „Protoplasmaaktivierung" auf, als welohe u. a. Stoffwechselbeschleunigung, Fieber, Hyperleukozytose, vermehrte Drüsensekretion, Lymphfluß, Anregung zur Produktion von Fermenten und Immunkörpern anzuselıell sınd. Diese Lehre, zuerst wenig Anklang findend, hat sich im Laufe der Jahre mehr und mehr durchgesetzt, da die Tatsachen eine eindringliche Sprache redeten.

Wenn man den obigen Leistungssteigerungen diejenigen Wirkungen vergleicht, die erfahrungsmäßig bei Kaltwasseranwendung eintreten: Leukozytose, Stoffwechsel-, Muskelkraft- und Sekretionsstejgerung usw., so ist zu erkennen, daB man in der Tat von einer Protoplasmaaktivierung als einem Erfolge der Kaltwasseranwendungen zu sprechen berechtigt ist. Und warum soll diese Aktivierung etwa rein auf nervöser Grundlage zustandekommen, warum soll micht dazu beitragen eine besondere Tätigkeit der Haut bzw. der Epidermis, bestehend in Sekretion von Stoffen, die gleich den parenteral eingefuhrten Eiweißsubstanzen stimulierend auf andere Organe wirken? So liegt meines Erachtens kein Grund vor, die Auffassung abzulehnen, daß bei der Reaktion durch den kalten Wasserreiz nach der obigen Richtung hin auf die Haut eingewirkt, das Zentralnervensystem - evtl. Im Sinne einer Antikenotoxinwirkung - zu erhohter Arbeit angeregt und somit das Erfrischungs- und erhöhte Leistungsgefühl hervorgerufen wird.

Die Betrachtungen über die Reaktion leiten unmittelbar über zu den abhärteıden Wassermaßnahmen, deren Erfolg wohl in den meisten Fällen nachweisbar in die Erscheinung tritt, die aber in ihrer bisherigen theoretischen Begründung und Deutung noch mehr als ein Fragezeichen übrig lassen.

Das Ziel der Abhärtung ist, den Organismus gegen krankmachende Einflusse von außen zu festigen und vor Infektionen zu schützen. Daß diese Festigung nur durch die prompte Reaktionsfähigkeit der Hautgefäße auf äußere Kaltreize erzielt wird, wie man bisher meist annalim, erscheint melir als zweifelliaft, und die Erzeugung bzw. Steigerung von Schutzstoffen in der Haut infolge Einwirkung kalter Wasserreize, zumal wenn letztere mit mechanischen Reizen vergesellschaftet sind, anzunehmen, erscheint mehr als anderes geeignet, das Wie und Warum der Abhärtung näher zu erklären.

Ich erinnere dabei an die Arbeit Keyssers ${ }^{2}$ ) über Erkältung, in der zum Ausdruck kommt, daß beim Tiere durch abhärtende $\mathrm{Ma} \beta$ nahmen die Schutzstoffe erheblich beeinflußt werden, so zwar, daß sie durch wiederholte Kältereize in ihrer Wirksamkeit uicht geschädigt werden: mit anderen Worten: die Abhärtung gegen Erkältung bedeutet ein Trainierell der Schutzstoffe in dem Sinne, daß die Erkältungseinflüsse diese Stoffe nicht meh herabsetzen - wie sie es bejm nicht abgehärteten tun - und damit den Organismus zu schädigen vermögen.

Dabei denkt Keys ser auch bereits an eine Wirkung der inineren Sekretion, die bestimmt ist, etwaige durch den ständigen Abbau auftretende giftige Substanzen - die die Schutzstoffe zu schädigen vermögen - zu entgiften, erwähnt jedoch nicht im einzelnen die in Betracht kommenden Organe, zu denen wir unter Anlehnung an die Hoffmannsche Auffassung die Haut zu rechnen berechtigt sind. Inwieweit gerade diese entgiftende Tätigkeit der Haut auch bei der Therapie verschiedener Stoftwechselkrankheiten mitzuwirken imstande ist, werden wir später sehen. - Dazu kommt, daß bei der Wechselwirkung der einzelnen Körperorgane aufeinander und der bereits erwähnten Fähigkeit des Körpers, daB die verschiedenen Orcane Stoffe an das Blut abgeben, die an irgendeiner Stelle im Organismus ganz bestimmte Prozesse auslösen (Abderhalden), infolge angeregter lebhafter Hauttätigkeit ein sekundärer Einfluß auf andere Organe ausgeübt wird, die ihrerseits dadurch wieder in verstärkte Aktion geraten. Und je mehr das Leben in den einzelnen Organen pulsiert, um so stärker werden sie durchblutet - um so resistenter werden sie gegen kraukınachende äußere Einflüsse werden, und um so siegreicher werden sie alle derartigen Angriffe überstehen. Wir sehen also, wie sich die Folgen der Abhärtung weit umfassender darstellen und wie tatsächlich Abhärtung und Kräftigung des Gesamtorganis mus innig und ursächlich zusammenhängen, nicht so sehr auf dem Wege der Gefäßreaktion und des Gefäßtrainings als auf dem einer inmersekretorischen Tätigkeit der Haut und ihrer Folgen.

Wenden wir uns nun zu der therapeutischen Seite der Hauttätigkeit: Hoffma nn erwähnt besonders den Einfluß der allgemeinen Lichtbäder und der Höhensonne auf den Gesamtorganismus und auf entfernte Krankheitsherde (z. B. bei innerer Tuberkulose), ferner den der Schwitzkuren auf eine Anzahll von Infektionskrankheiten, wobel er nicht nur eine Entlastung des Organismus von Schlackenst offen, sondern auch die Erze ugung von Schutz s tof fen auniunmt. Auch den günstigen Einflub der systematischen Schmierseifen- und Quecksılbereinreibungen hebt er hervor, denen meines Erachtens noch die Eilreibungen von Kreolin und Terpentin bei chronisclıcn Lungenkatarrlıeı hinzuzufügen wären, wie auch in besonderein Maße die hydrotherapeutischen Anwendungen bei chronischen, z. B. Lungentuberkulose und akuten Infektionskrankheiten Nehmen wir zunächst die kuhlen und kalten Bäder beim Typhus. Nach ihnen tritt fast regelmäßig nicht nur ein verschieden starker Abfall der Temperatur ein, sondern das Sensorium hellt sich auf, der Puls bessert sich und die Appetenz steigt. Gewiß sprechen die durch das kalte Wasser erzeugten Reize auf Nerven- und Zirkulationssystem ein gewichtiges Wort mit. Aber nicht nur die symptomatischen Ertolge, sondern auch die den ganzen Krankheitsablauf bereinflussenden Nachwirkungen dieser $B$ äder, ebenso wie auch die der oftmals wiederholten, die Körpertemperatur jeweilig nur unwesentlich herabsetzenden kalten feil abwaschungen, sind doch in vielen Fällen so evident, daß in der Tat noch andere Kräfte und Vorgänge usw. zur Erklärullg solcher Besserungen angenommen werden müssen. Durch die vorausgesetzte Fähigkeit der Haut, eine esophylaktische Tätigkeit bei genügender Reizung auszuüben und Schutzstoffe zu bilden, wird nunmehr in der Tat das Verständ nis für die genannten Erfolge der Hydrotherapie bei Typhus und anderen Infektionskrankheiten wesentlich erleichtert und ge fördert. In gleicher Weise ist es nun erlaubt, auch bei den Stammpackungen, neben dem EinfluB auf Zirkulation und Nervensystem, auch noch einen solchen auf die Erzeugung von Abwehrstoffen in der Haut als Ursache für ihre günstige Wirkung bei den akuten Infektionskrankheiten besonders der Kinder (man denke nur an die manchmal erstaunlich schnelle und günstige Wirkung beim Scharlach, bei den Masern, bei der Diphtherie usw.!) anzunehmen.

Während die Stammpackungen kalt oder kühl angelegt - ihre Wirkung wird oft noch durch Alkoholzusatz zum Wasser verstärkt die Bäder bei Typhus und Pneumonie mit einer Anfangstemperatur von $5-6^{\circ}$ unter der augenblicklichen Körperwärme gegeben und weiterliin noch abgekühlt werden, hat man anderseits auch die gute Wirkung heißer Bäder bei Cholera und Meningitis cerebrospinalis in vielen Fällen erprobt und bewährt gefunden. Auch hier wird man neber den anderen Einflüssen auch einen solchen auf die esophylaktische Eigenschaft der Haut annehmen und damit den Schluß wagen dürfen, daß entweder jede stärkere thermische Reizung der Haut gleich ob warm oder kalt, eine esophylaktische Tätigkeit der Epithermisschicht erweckt, oder daß bei der einen Krankheit die spezifischen Schutzstoffe auf hyperthermische, bei der auderen dagegen auf hypothermische Reize hin erzeugt, vermehrt und in den Kreislauf abgeführt werden ${ }^{1}$ ).

Die von Hoffmann hervorgehobene besonders gute Wirkung der Quecksilbereinreibungen auf die Haut in Verbindung mit den Aachen-Burtscheider Bädern bei der Syphilis ist meinęs Erachtens aus mehreren Komponenten zusammengesetzt. Neben der Einverleibung des Quecksilber's konimt in Aachen-B. einmal die Bearbeitung der Haut bei der Einreibung selbst in Betracht, ferner aber auch die gleichzeitige Verabreichung von warmen oder heißen Bädern. Durch sie werden

1) $\mathrm{DaB}$ auch heiße Bäder erfrischend und abhärtend wirken können, ist eine bekannte Tatsache, die besonders durch die seit Alters von den Japanern zu diesem Zwecke angewanden Bäder von $40-42^{\circ} \mathrm{C}$ treffend illustriert wird. 
nicht nur die Poren geöffnet und damit dem Eindringen der eingeriebenen $\mathrm{Hg}$.Teilchen der Boden bereitet, sondern auch die Haut iil einer Weise gereizt, daB eine esophylaktische Wirkung die Folge ist und durch sie die günstigen therapeutischen Ertolge dieser Inunktionskuren mehr minder verstärkt werden.

Wie weit dabei allein die Wassertemperaturen mitwirken oder etwa noch die chemische usw. Beschaffenheit des Thermalwassers zu bewerten ist, muß späteren Untersuchungen vorbehaiten bleiben. lmmerhin dirften auch bei dem Heer der vielen anderen Krankheiten, für die die Patienten in Heilbädern Genesung oder Besserung suchen und auch finden, eine Anregung der Hauttätigkeit durch die Beschaffenheit des Badewassers und Erzeugung einer Esophylaxie sehr wohl möglich sein.

Die Wirkung der Mineralwässer bei ihrer äußeren Anwendung wurde bisher daningehend erklärt, daß man ein Verbleiben $z$. B. von Salzteilchen des Wassers auf der Oberfläche der Haut bzw. in ihren Poren annahm und eine stärkere Durchblutung der Körperoberfläche auf die Nachwinkung dieser Salzteilchen zuruckführte. Dabes machen nach Glax Chlornatrium-, Chlorkalium- oder Chlorkalziumbäder keinen Unterschied untereinander und beeinflussen indifferente warme Salzbäder weder die Wärmeabgabe, noch das Tastgcfühl, noch die Puls- und Respirationsfrequenz; und auch der respiratorische Gaswechsel wird nicht oder nur selır wenig durch sie verändert. Und doch treten erfahrungsmäßig nach solcnen Bädern, wozu ja auch (mehr weniger) unsere Burtscheider Bäder zu rechnen sind, Wirkungen ein, die schieślich nicht $\mathrm{nur}$ auf den i.1 den $10 \mathrm{u}-1000$ Liter fassenden Wannen vermehrten Wasserdruck zurückzuführen sein dürften, wenngleich er naturg cmäß bei der Erklärung der Heilwirkung mitspricht. - In der Tat liegt die Folgerung nicht so ganz ferne, auch bei den Heilbädern, selbst wenn sie indifferente jemperaturen zeigen und somit nur durch ihre chemische bzw. elektrische Beschaffenheit wirken können, einen durch diese Beschaffenlieit ausgeübten Reiz auf die Haut anzunchmen, der dazu dient, eine Art ilmnersekretorischer Tätigkeit der Haut hervorzurufen. Und nichts hindert, meines Erachtens, außer der Erzeugung von Sclutzstoffen auch die von Fermenten und Hormonen in der gereizten Haut anzuiiehmen; gehören doch auch diese Substanzen zu denjenigen, deren Auftreten als Ausdruck einer allgemeinen Lcistungssteigerung durch Protoplasmaaktivierung aufzufassen ist; einer Stcigerung, die nicht nur nach der parenteralen Einverleibung von Eiweißspaltprodukten, sondern sogar schon mach der von Kochsalz eintreten kann. Das Auftreten und die Mitarbeit gerade von Fermenten und Hormonen bei entsprechender Einwirkung der Heilbäder auf die Haut würden ungemein viel zum Verständnis der guten Wirkung der Kochsalz-, Schwefel-, Jod-, Radium- usw. Bäder, z. B. bei gichtischen und diabetischen, chlorotischen und anderen Erkrankungen, beizutragen vermögen. Denn ich glaube nicht, daß die Trinkkur allein dic genannten Krankheiten maßgebend beeinflußt, sondern erachte die Verabreichung von Mineralbäderı für (iıen ganz wesentl.chon Teil dcr Badekur. Dieser Auffassung sind $u$. a. offenbar auch $M$ a a s $e^{1}$ ) und Saalecker gewesen, die bei ihrer eingehenden Arbeit über den Einfluß der Neuenahrer Quellen auf den Diabetcs mellitus die untersuchten und beobachteten Patienten nicht nur trinken, sondern auch methodisch baden ließen.

Nur noch einige Worte zur Lichttherapie.

Hof $f m a n n$ erwähnt die unverkennbar gute Wirkung der Lichtbäder (so auch der künstlichen Höhensonnc usw.) auf die Tuberkulose und erklärt sie sich ebenfalls durch die esophylaktische Fäligkeit der Haut, wie ja auch Grau früher schon die Entstehung von Abwehrstoffen bei der Anwendung der Quarzlanpe annahm, die in der bestrahlten Haut, in Wecliselwirkung mit dem Krankheitsherde, gebildet werden sollten ${ }^{2}$ ). lch möchte noch zwel weitere Krankheiten antühren, bei denen besonders die allgemeinen Gluhhlichtbäder häufig ganz auffallende Erfolge erzielen. Das sind die Pubertitschlorose und das Asthma bronchiale. Bei beiden Krankheiten erklärte man sich bisher überwiegend die gute Wirkung mit der Diaphorese bzw. der Steigerung des Stoffwechsels, wenn auch v. Strü mpell das Vorliegen einer spezifischen Wirkung auf das Asthma für möglich hielt. Die ganze Frage erhält ein anderes Gesicht mit dem Augenblick, wo eine spezifische Wirkung der wärmenden Lichtstrahlen auf die Haut als walırscheinlich anzunehmen und dic Beeinflussung entsprechender endokriner Drüsen, wie Nebennieren, Keimdrüsen usw., von ihr aus etwa in Rechnung $\mathrm{zu}$ stellen sind. Gerade das Beispiel dieser beiden Erkrankungen scheint mir recht wohl geeignet, zur Unterstützung der Anschauungen von der innersekreiorischen Tätigkeit der Haut zu dienen: denı der Zusammenhang des Asthma bronchiale und der l'ubertätschlorose mit der Funktion endokriner Drüsen ist recht wahrscheinlich und infolgcdessen dic Schlußfolgerung nicht so fernliegend, daß, wenn die durch dic Lichtbäder getätigten Hautreize imstande sind, die obigen Krankheiten günstig zu bceinflussen, dies zu einem verschieden hohen Maße geschieht durch die Erzeugung usw. entsprechender auf die genannten Drüsen wirkender Stoffe bzw. Hormone. Oder man könnte auch glauben, daß in der Haut Substanzen gebildet werden, die die betreffenden Krankheitsprozesse selbst zu beeinflussen vermögen, und sei es nur dadurch, daß sie cine entgiftende Wirkung ausüben. Dabei sollen die Wahrscheinlichkeit der Stoffwechselfördcrung durch die Esophylaxie der Haut und etwaige nervöse Wirkungen durch die Lichtbäder keineswegs als belanglos angesehen werden.

1) Zschr. f. Baln. 1915 Nr. 7, 8 und 9. - s) M.m. W. 1917 Nr. 48
Den elektrischen Glühlichtbädern glatıbte man bislang nur insofern eine Sonderstellung vor den Heißluftbädern einrä̉umen zu müssen, als in ihnen wegen der relativ schnellen und tief in die Gewebe eindringenden leuchtenden und wärmenden Strahlen des Glühlichts die Schweißabsonderung in kürzester Frist und bei niedrigerer Temperatur als in den Heißluftkästen eintritt. Den chemisch und bakteriell wirksamen, kurzwelligen Strahlen - blau, violett, ultraviolett -, die nur in verschwindender Menge im Glülllicht vorhanden sind, kann dagegen schon deswegen kcin größerer Einfluß zugebilligt werden, weil sie bereits in den obersten Hautschichten (Epıdermıs, Rete papillare) fast vóltig absorbiert werden. Liese Sonderstellung der Glühlichtbäder würde nunmehr noch eine Steigerung erfahren durch die Annahme, daß die Haut in ihnen infolge der schneller in die Tiefe dringenden Wärmestrahlen auch schneller zu einer innersekretorischen Tätigkeit an. geregt wird als durch die in den HeiBluftbädern wirksame, gele it e te Wärme.

Im Zusammenhang mit dieser Betrachtung würde auch noch kurz der Beeinflussung der Hauttätigkeit durch die Sonnenbäder zu gedenken sein. Nicht nur die Tuberkulose der inneren und Bewegungsorgane usw., sondern auch eine Reihe anderer chronischer Krankheiten, z. B. Malaria, Lues, Polyartlıritis, Chlorose, Anämic, wird bekanntlich durch die Anwendung von Sonnenbädern auf das günstigste beeinflußt. Hier liegt die Erklärung der Erfolge nach obigen Betrachtungen besonders nahe und offenbar, wobei anderseits die schweißtreibende Wirkung natürlich nicht außerachtgelassen werden darf.

Es wäre leicht, noch manche andre physikalisch-therapeutische Prozedur, so das Luftbad, die Massage usw., hier anzuführen und ihre Beziehung zur Anregung der mehrfach genannten Hauttätigkeit durchzusprechen.

Aber ich meine, daß die obigen Ausführungen hinreichen, um eine Vorstellung zu geben von dem Zusammenhang der innersekretorischen Hauttatigkeit und hydriatischen sowie anderer physikalischtherapeutischen Anwendungen und da $\beta$ sie ferner geeignet silnd, zur Erklärung der mit ilınen erzieiten Erfolge in etwas beizutragen.

$\mathrm{Nachschrift.} \mathrm{Die} \mathrm{postalischen} \mathrm{Verhältnisse} \mathrm{im} \mathrm{besetzten} \mathrm{Ge-}$ biet haben es mit sich gebracht, daß ich erst nach Abschluß vorstehender Arbeit den Aufsatz von Weichardt in Nr. 4 der M. m. W. 1920 „Ueber unspezifische Leistungssteigerung“ zu Gesicht bekam. Ich freue mich, feststellen zu können, daß Weich a rdt - wenn auch nur in aller Kürze - ähnliche Betrachtungen im AnschluB an die Hof $f \mathrm{~m}$ an $n$ sche Arbeit anstellt über die Beeinflussung der Haut im Sinne der Protoplasmaaktivierung und daß auch er die Euphoric und gesteigerte Leistungsfähigkeit nach einer physikalischen Behandlung, wie es die Faradisierung uach Bergonié darstellt, ansieht als die Folge derselben Protoplasmaaktivierung. 